\title{
STABILITY OF MAPPINGS ON MULTI-NORMED SPACES
}

\author{
H. G. DALES \\ Department of Pure Mathematics, University of Leeds, Leeds LS2 9JT, United Kingdom \\ e-mail: garth@maths.leeds.ac.uk \\ and MOHAMMAD SAL MOSLEHIAN \\ Department of Mathematics, Ferdowsi University, P.O. Box 1159, Mashhad 91775, Iran. \\ e-mail:moslehian@ferdowsi.um.ac.ir
}

(Received 1 September, 2006; revised 13 December, 2006; accepted 9 January, 2007)

DEDICATED TO THE MEMORY OF M. E. POLYAKOV

\begin{abstract}
In this paper, we define multi-normed spaces, and investigate some properties of multi-bounded mappings on multi-normed spaces. Moreover, we prove a generalized Hyers-Ulam-Rassias stability theorem associated to the Cauchy additive equation for mappings from linear spaces into multi-normed spaces.
\end{abstract}

2000 Mathematics Subject Classification. Primary 39B82; Secondary 39B52, 46B99, 47A99.

1. Introduction. In 1940, Ulam [22] first raised the stability problem of functional equations: 'For which metric groups $G$ is it true that an $\varepsilon$-automorphism of $G$ is necessarily near to an automorphism?' In the next year, Hyers [9] gave a partial affirmative answer to the question of Ulam in the context of Banach spaces, and in 1978, Th. M. Rassias [19] proved the following theorem, which subsumes Hyers' theorem in the case where $p=0$.

Let $E$ and $F$ be real normed spaces with $F$ complete, let $f: E \rightarrow F$ be a mapping such that, for each fixed $x \in E$, the mapping $t \mapsto f(t x)$ is continuous on $\mathbb{R}$, and let $\varepsilon \geq 0$ and $p \in[0,1)$ be such that

$$
\|f(x+y)-f(x)-f(y)\| \leq \varepsilon\left(\|x\|^{p}+\|y\|^{p}\right) \quad(x, y \in E) .
$$

Then there exists a unique linear mapping $T: E \rightarrow F$ such that

$$
\|f(x)-T x\| \leq \varepsilon\|x\|^{p} /\left(1-2^{p-1}\right) \quad(x \in E) .
$$

In 1990, Th. M. Rassias [20] asked whether such a theorem can also be proved for $p \geq 1$. In 1991, Gajda [7], following the same approach as in [19], gave an affirmative solution to this question when $p>1$, but it was proved by Gajda [7] and Rassias and Šemrl [21] that one cannot prove an analogous theorem when $p=1$. The result of Rassias, which is also true for $p<0$ (where we adopt the convention that $\|0\|^{p}=\infty$ ), has influenced the development of what is now called the Hyers-Ulam-Rassias stability phenomenon. In 1994, a generalization of Rassias' theorem, the so-called generalized Hyers-Ulam-Rassias stability theorem, was obtained by Găvruta [8]: he replaced the bound $\varepsilon\left(\|x\|^{p}+\|y\|^{p}\right)$ in (1.1) by a general control function $\varphi(x, y)$. 
During the last decades several stability problems of functional equations have been investigated by a number of mathematicians $[3,5, \mathbf{1 0}, \mathbf{1 5}, \mathbf{2 0}]$. These results have many applications in information theory, physics, economic theory and social and behavioural sciences $[\mathbf{1}, \mathbf{2}]$. We briefly mention some applications within the theory of Banach algebras. In each case a key step is the use of an analogue of our Theorem 3.5.

About 20 years ago, papers appeared dealing with perturbation theory: they asked when is it true that a mathematical object which satisfies a certain property approximately is necessarily close to something which satisfies the property exactly. Many of these result are described in [12]. For example, let $A$ be a Banach algebra, and let $T: A \rightarrow \mathbb{C}$ be a linear functional such that

$$
|T(a b)-T(a) T(b)|<\delta|T(a)||T(b)| \quad(a, b \in A) .
$$

Then $\|T\| \leq 1+\delta$. Next, let $A$ and $B$ be subalgebras of a Banach algbera $C$ such that $A$ and $B$ are 'geometrically close'. Then it often follows (see [12]) that $A$ and $B$ are isomorphic. Similarly, let a Banach space $A$ have two multiplications that make it into an algebra and are such that the two products are close as bilinear maps. Then the two algebras so formed often have common algebraic properties. These ideas are related to that of approximately multiplicative maps, as studied by Johnson in [13] and [14]. A pair $(A, B)$ of Banach algebras is AMNM if, for each $\varepsilon>0$ and each $K>0$, there exists $\delta>0$ such that, for each bounded linear map $T: A \rightarrow B$ such that $\|T\| \leq K$ and $\|T(a b)-T(a) T(b)\| \leq \delta\|a\|\|b\|$ for all $a, b \in A$, there is a bounded homomorphism $\theta: A \rightarrow B$ such that $\|T-\theta\| \leq \varepsilon$. The theorems of Johnson discuss which Banach algebras are AMNM; the proofs and conditions involve the cohomology theory of Banach algebras. Related matters involving approximate cohomology groups and approximate amenability are discussed in [18].

In this paper, following [6], we define multi-normed spaces, and investigate some properties of multi-bounded mappings on multi-normed spaces. Moreover, we prove a generalized Hyers-Ulam-Rassias stability theorem associated to the Cauchy additive equation for mappings from linear spaces into multi-normed spaces by using a fixed point approach in the style of $[4, \mathbf{1 1}, \mathbf{1 7}]$. The theory of multi-normed spaces and of multi-Banach algebras originated in [6].

Our expectation is that our Theorem 3.5 will have similar applications to the above in the theory of multi-normed spaces and algebras.

Let $(E,\|\cdot\|)$ be a complex linear space, and let $k \in \mathbb{N}$. We denote by $E^{k}$ the linear space $E \oplus \ldots \oplus E$ consisting of $k$-tuples $\left(x_{1}, \ldots, x_{k}\right)$, where $x_{1}, \ldots, x_{k} \in E$. The linear operations on $E^{k}$ are defined coordinatewise. When we write $\left(0, \ldots, 0, x_{i}, 0, \ldots, 0\right)$ for an element in $E^{k}$, we understand that $x_{i}$ appears in the $i^{\text {th }}$ coordinate. The zero element of either $E$ or $E^{k}$ is denoted by 0 . We denote by $\mathbb{N}_{k}$ the set $\{1,2, \ldots, k\}$ and by $\mathfrak{S}_{k}$ the group of permutations on $k$ symbols; we set $\mathbb{T}=\{z \in \mathbb{C}:|z|=1\}$.

2. Multi-normed spaces and multi-bounded operators. We start this section by recalling the notion of a multi-normed space from [6]. Throughout this section $(E,\|\cdot\|)$ denotes a complex normed space.

DefinITION 2.1. A multi-norm on $\left\{E^{n}: n \in \mathbb{N}\right\}$ is a sequence

$$
\left(\|\cdot\|_{n}\right)=\left(\|\cdot\|_{n}: n \in \mathbb{N}\right)
$$


such that $\|\cdot\|_{n}$ is a norm on $E^{n}$ for each $n \in \mathbb{N}$, such that $\|x\|_{1}=\|x\|$ for each $x \in E$, and such that the following axioms are satisfied for each $n \in \mathbb{N}$ with $n \geq 2$ :

$$
\begin{gathered}
\left\|\left(x_{\sigma(1)}, \ldots, x_{\sigma(n)}\right)\right\|_{n}=\left\|\left(x_{1}, \ldots, x_{n}\right)\right\|_{n} \quad\left(\sigma \in \mathfrak{S}_{n}, x_{1}, \ldots, x_{n} \in E\right) ; \\
\left\|\left(\alpha_{1} x_{1}, \ldots, \alpha_{n} x_{n}\right)\right\|_{n} \leq\left(\max _{i \in \mathbb{N}_{n}}\left|\alpha_{i}\right|\right)\left\|\left(x_{1}, \ldots, x_{n}\right)\right\|_{n} \quad\left(\alpha_{1}, \ldots, \alpha_{n} \in \mathbb{C}, x_{1}, \ldots, x_{n} \in E\right) ; \\
\left\|\left(x_{1}, \ldots, x_{n-1}, 0\right)\right\|_{n}=\left\|\left(x_{1}, \ldots, x_{n-1}\right)\right\|_{n-1} \quad\left(x_{1}, \ldots, x_{n-1} \in E\right) ; \\
\left\|\left(x_{1}, \ldots, x_{n-1}, x_{n-1}\right)\right\|_{n}=\left\|\left(x_{1}, \ldots, x_{n-1}\right)\right\|_{n-1} \quad\left(x_{1}, \ldots, x_{n-1} \in E\right) .
\end{gathered}
$$

In this case, we say that $\left(\left(E^{n},\|\cdot\|_{n}\right): n \in \mathbb{N}\right)$ is a multi-normed space.

Motivations for the study of multi-normed spaces (and multi-normed algebras) and many examples are given in [6].

Suppose that $\left(\left(E^{n},\|\cdot\|_{n}\right): n \in \mathbb{N}\right)$ is a multi-normed space. The following properties are almost immediate consequences of the axioms. First

$$
\|(x, \ldots, x)\|_{k}=\|x\| \quad(k \in \mathbb{N}, x \in E) .
$$

Secondly, let $j, l \in \mathbb{N}$ with $j \leq l$, and let $x_{1}, \ldots, x_{j}, y_{1}, \ldots, y_{l} \in E$ be such that $\left\{x_{1}, \ldots, x_{j}\right\}$ is a subset of $\left\{y_{1}, \ldots, y_{l}\right\}$. Then

$$
\left\|\left(x_{1}, \ldots, x_{j}\right)\right\|_{j} \leq\left\|\left(y_{1}, \ldots, y_{l}\right)\right\|_{l} .
$$

Thirdly, we have

$$
\max _{i \in \mathbb{N}_{k}}\left\|x_{i}\right\| \leq\left\|\left(x_{1}, \ldots, x_{k}\right)\right\|_{k} \leq \sum_{i=1}^{k}\left\|x_{i}\right\| \leq k \max _{i \in \mathbb{N}_{k}}\left\|x_{i}\right\| \quad\left(k \in \mathbb{N}, x_{1}, \ldots, x_{k} \in E\right) .
$$

It follows from (c) that, if $(E,\|\cdot\|)$ is a Banach space, then $\left(E^{n},\|\cdot\|_{n}\right)$ is a Banach space for each $n \in \mathbb{N}$; in this case $\left(\left(E^{n},\|\cdot\|_{n}\right): n \in \mathbb{N}\right)$ is a multi-Banach space.

Now we recall three important examples of multi-norms for an arbitrary normed space $E$; see [6] for details and many other examples.

EXAmple 2.2. The sequence $\left(\|\cdot\|_{n}: n \in \mathbb{N}\right)$ on $\left\{E^{n}: n \in \mathbb{N}\right\}$ defined by

$$
\left\|\left(x_{1}, \ldots, x_{n}\right)\right\|_{n}:=\max _{i \in \mathbb{N}_{n}}\left\|x_{i}\right\| \quad\left(x_{1}, \ldots, x_{n} \in E\right)
$$

is a multi-norm called the minimum multi-norm. The terminology 'minimum' is justified by property (c).

ExAmple 2.3. Let $\left\{\left(\|\cdot\|_{n}^{\alpha}: n \in \mathbb{N}\right): \alpha \in A\right\}$ be the (non-empty) family of all multinorms on $\left\{E^{n}: n \in \mathbb{N}\right\}$. For $n \in \mathbb{N}$, set

$$
\left\|\left(x_{1}, \ldots, x_{n}\right)\right\|\left\|_{n}:=\sup _{\alpha \in A}\right\|\left(x_{1}, \ldots, x_{n}\right) \|_{n}^{\alpha} \quad\left(x_{1}, \ldots, x_{n} \in E\right) .
$$

Then $\left(\||\cdot|\|_{n}: n \in \mathbb{N}\right)$ is a multi-norm on $\left\{E^{n}: n \in \mathbb{N}\right\}$, called the maximum multinorm.

EXAmPle 2.4. Let $(E,\|\cdot\|)$ be a Banach lattice, and define

$$
\left\|\left(x_{1}, \ldots, x_{n}\right)\right\|_{n}:=\left\|\left|x_{1}\right| \vee \ldots \vee\left|x_{n}\right|\right\| \quad\left(x_{1}, \ldots, x_{n} \in E\right) .
$$


Then $\left(\|\cdot\|_{n}: n \in \mathbb{N}\right)$ is a multi-Banach space.

Definition 2.5. Let $\left(\left(E^{k},\|\cdot\|_{k}\right): k \in \mathbb{N}\right)$ be a multi-normed space. A sequence $\left(x_{n}\right)$ in $E$ is a multi-null sequence if, for each $\varepsilon>0$, there exists $n_{0} \in \mathbb{N}$ such that

$$
\sup _{k \in \mathbb{N}}\left\|\left(x_{n}, \ldots, x_{n+k-1}\right)\right\|_{k}<\varepsilon \quad\left(n \geq n_{0}\right) .
$$

Let $x \in E$. Then

$$
\operatorname{Lim}_{n \rightarrow \infty} x_{n}=x
$$

if $\left(x_{n}-x\right)$ is a multi-null sequence; in this case, the sequence $\left(x_{n}\right)$ is multi-convergent to $x$ in $E$.

Definition 2.6. Let $\left(\left(E^{k},\|\cdot\|_{k}\right): k \in \mathbb{N}\right)$ and $\left(\left(F^{k},\|\cdot\|_{k}\right): k \in \mathbb{N}\right)$ be multi-normed spaces, and let $x \in E$. A mapping $f: E \rightarrow F$ is multi-continuous at the point $x$ if $\operatorname{Lim}_{n \rightarrow \infty} f\left(x_{n}\right)=f(x)$ in $F$ whenever $\operatorname{Lim}_{n \rightarrow \infty} x_{n}=x$ in $E$.

Now we give the definition from [6] of a multi-bounded operator between multinormed spaces; this is similar to the notion of bounded linear operator between two normed spaces.

Definition 2.7. Let $\left(\left(E^{k},\|\cdot\|_{k}\right): k \in \mathbb{N}\right)$ and $\left(\left(F^{k},\|\cdot\|_{k}\right): k \in \mathbb{N}\right)$ be multi-normed spaces. A linear operator $T: E \rightarrow F$ is multi-bounded if

$$
\|T\|_{m b}:=\sup _{n} \sup \left\{\frac{\left\|\left(T x_{1}, \ldots, T x_{n}\right)\right\|_{n}}{\left\|\left(x_{1}, \ldots, x_{n}\right)\right\|_{n}}:\left(x_{1}, \ldots, x_{n}\right) \neq 0\right\}<\infty .
$$

The collection of multi-bounded operators from $E$ to $F$ is denoted by $\mathcal{M}(E, F)$. It is easy to see that $\left(\mathcal{M}(E, F),\|\cdot\|_{m b}\right)$ is a Banach subspace of the Banach space $\mathcal{B}(E, F)$ of all bounded linear operators from $E$ to $F$, and that $\mathcal{M}(E, F)$ contains all nuclear operators. In fact, $\mathcal{M}(E, F)$ is also a multi-Banach space in a canonical way; see [6, Chapter 4].

THEOREM 2.8. Let $\left(\left(E^{k},\|\cdot\|_{k}\right): k \in \mathbb{N}\right)$ and $\left(\left(F^{k},\|\cdot\|_{k}\right): k \in \mathbb{N}\right)$ be multi-normed spaces. Then a linear mapping $T: E \rightarrow F$ is multi-continuous if and only if it is multibounded.

Proof. Suppose that $T$ is multi-bounded, and let $\left(x_{n}\right)$ be a multi-null sequence in $E$. Then, for each $\varepsilon>0$, there exists $n_{0} \in \mathbb{N}$ such that

$$
\sup _{k \in \mathbb{N}}\left\|\left(x_{n}, \ldots, x_{n+k-1}\right)\right\|_{k}<\varepsilon \quad\left(n \geq n_{0}\right) .
$$

But now

$$
\sup _{k \in \mathbb{N}}\left\|\left(T x_{n}, \ldots, T x_{n+k-1}\right)\right\|_{k} \leq\|T\|_{m b} \varepsilon \quad\left(n \geq n_{0}\right)
$$

and so $\left(T x_{n}\right)$ is a multi-null sequence in $F$. Thus $T$ is multi-continuous.

Conversely, suppose that $T$ is multi-continuous and assume that $T$ is not multibounded. Then, for each $n \in \mathbb{N}$, there exists a positive integer $k_{n} \geq n$ such that

$$
\sup \left\{\frac{\left\|\left(T x_{1}, \ldots, T x_{k_{n}}\right)\right\|_{k_{n}}}{\left\|\left(x_{1}, \ldots, x_{k_{n}}\right)\right\|_{k_{n}}}:\left(x_{1}, \ldots, x_{k_{n}}\right) \neq 0\right\}>n^{2} .
$$


Hence there exists $\left(y_{1, n}, \ldots, y_{k_{n}, n}\right) \in E^{k_{n}}$ such that

$$
\frac{\left\|\left(T y_{1, n}, \ldots, T y_{k_{n}, n}\right)\right\|_{k_{n}}}{\left\|\left(y_{1, n}, \ldots, y_{k_{n}, n}\right)\right\|_{k_{n}}}>n^{2} .
$$

Now set

$$
x_{j, n}=\frac{y_{j, n}}{n^{2}\left\|\left(y_{1, n}, \ldots, y_{k_{n}, n}\right)\right\|_{k_{n}}} \quad\left(j \in \mathbb{N}_{k_{n}}\right)
$$

Then

$$
\left\|\left(x_{1, n}, \ldots, x_{k_{n}, n}\right)\right\|_{k_{n}}<\frac{1}{n^{2}} \quad \text { and } \quad\left\|\left(T x_{1, n}, \ldots, T x_{k_{n}, n}\right)\right\|_{k_{n}}>1 .
$$

Consider the sequence

$$
\left(z_{i}\right)=\left(x_{1,1}, \ldots, x_{k_{1}, 1}, x_{1,2}, \ldots, x_{k_{2}, 2}, \ldots, x_{1, n}, \ldots, x_{k_{n}, n}, \ldots\right)
$$

in $E$. We claim that the sequence $\left(z_{i}\right)$ is multi-null in $E$. Indeed, given $\varepsilon>0$, there exists $j \in \mathbb{N}$ with $j \geq 2$ such that $\sum_{i=j}^{\infty} 1 / i^{2}<\varepsilon$, and then

$$
\begin{aligned}
\left\|\left(x_{1, j}, \ldots, x_{k_{j}, j}, \ldots, x_{1, j+n}, \ldots, x_{k_{j+n}, j+n}\right)\right\|_{k_{j}+\ldots+k_{j+n}} & \leq \sum_{\ell=j}^{j+n} \|\left(x_{1, \ell}, \ldots, x_{k_{\ell}, \ell} \|_{k_{\ell}}\right. \\
& \leq \sum_{\ell=j}^{j+n} \frac{1}{\ell^{2}}<\varepsilon \quad(n \in \mathbb{N}) .
\end{aligned}
$$

Fix $n_{0}=k_{1}+\cdots+k_{j-1}+1$. For $n, k \in \mathbb{N}$ with $n \geq n_{0}$, choose $m \in \mathbb{N}$ with

$$
k_{j}+\cdots+k_{j+m}>\left(n-k_{1}-\cdots-k_{j-1}\right)+k-1 \text {. }
$$

Then $\left\{z_{n}, \ldots, z_{n+k-1}\right\} \subseteq\left\{x_{1, j}, \ldots, x_{k_{j}, j}, \ldots, x_{1, j+m}, \ldots, x_{k_{j+m}, j+m}\right\}$, and so it follows from (2.1) and property (b) that

$$
\left\|\left(z_{n}, \ldots, z_{n+k-1}\right)\right\|_{k} \leq\left\|\left(x_{1, j}, \ldots, x_{k_{j}, j}, \ldots, x_{1, j+m}, \ldots, x_{k_{j+m}, j+m}\right)\right\|_{k_{j}+\ldots+k_{j+m}} \leq \varepsilon .
$$

Hence $\sup _{k \in \mathbb{N}}\left\|\left(z_{n}, \ldots, z_{n+k-1}\right)\right\|_{k} \leq \varepsilon\left(n \geq n_{0}\right)$, and so $\left(z_{i}\right)$ is multi-null. The sequence $\left(T z_{i}\right)$ is clearly not a multi-null sequence in $F$, and so $T$ is not multicontinuous, giving the required contradiction.

3. Generalized Hyers-Ulam-Rassias stability. We begin this section with 'the alternative fixed point theorem' [16]. A generalized metric space is a pair $(X, d)$, where $X$ is a non-empty set and $d: X \times X \rightarrow[0, \infty]$ satisfies the usual axioms. A map $J$ : $X \rightarrow X$ is strictly contractive if there exists a 'Lipschitz constant' $0 \leq L<1$ such that $d(J x, J y) \leq L d(x, y) \quad(x \in X)$.

THEOREM 3.1. Let $(X, d)$ be a complete generalized metric space, and let $J: X \rightarrow X$ be a strictly contractive mapping with a Lipschitz constant $L<1$. Then, for each element $x \in X$, either

(F1) $d\left(J^{n} x, J^{n+1} x\right)=\infty \quad(n \geq 0)$, or

(F2) there exists $n_{0} \geq 0$ such that: 
(F20) $d\left(J^{n} x, J^{n+1} x\right)<\infty \quad\left(n \geq n_{0}\right)$;

(F21) the sequence $\left(J^{n} x\right)$ converges to a fixed point $y^{*}$ of $J$;

(F22) $y^{*}$ is the unique fixed point of $J$ in the set $U=\left\{y \in X: d\left(J^{n_{0}} x, y\right)<\infty\right\}$;

(F23) $d\left(y, y^{*}\right) \leq d(y, J y) /(1-L) \quad(y \in U)$.

The following lemma gives a useful strictly contractive mapping. We adopt the convention that inf $\emptyset=\infty$.

LemmA 3.2. Let $k \in \mathbb{N}$, and let $E$ and $F$ be linear spaces such that $\left(F^{k},\|\cdot\|\right)$ is a Banach space. Suppose that there exist $0 \leq M<1, \lambda>0$, and a map $\psi: E^{k} \rightarrow[0, \infty)$ such that

$$
\psi\left(\lambda x_{1}, \ldots, \lambda x_{k}\right) \leq \lambda M \psi\left(x_{1}, \ldots, x_{k}\right) \quad\left(x_{1}, \ldots, x_{k} \in E\right)
$$

Set $X:=\{g: E \rightarrow F: g(0)=0\}$, and define $d: X \times X \rightarrow[0, \infty]$ by

$$
\begin{aligned}
d(g, h)=\inf \{c>0: & \left\|\left(g\left(x_{1}\right)-h\left(x_{1}\right), \ldots, g\left(x_{k}\right)-h\left(x_{k}\right)\right)\right\| \\
& \left.\leq c \psi\left(x_{1}, \ldots, x_{k}\right) \text { for all } x_{1}, \ldots, x_{k} \in E\right\} .
\end{aligned}
$$

Then $(X, d)$ is a complete generalized metric space, and the mapping $J: X \rightarrow X$ defined by $(J g)(x):=g(\lambda x) / \lambda \quad(x \in X)$ is a strictly contractive mapping.

Proof. It is easy to see that $(X, d)$ is complete generalized metric space.

For elements $g, h \in X$ with $d(g, h)<\infty$, take $c>d(g, h)$. Then, for each $x_{1}, \ldots, x_{k} \in E$, we have $\left\|\left(g\left(x_{1}\right)-h\left(x_{1}\right), \ldots, g\left(x_{k}\right)-h\left(x_{k}\right)\right)\right\| \leq c \psi\left(x_{1}, \ldots, x_{k}\right)$, and so

$$
\begin{aligned}
\left\|\left(\frac{1}{\lambda} g\left(\lambda x_{1}\right)-\frac{1}{\lambda} h\left(\lambda x_{1}\right), \ldots, \frac{1}{\lambda} g\left(\lambda x_{k}\right)-\frac{1}{\lambda} h\left(\lambda x_{k}\right)\right)\right\| & \leq \frac{1}{\lambda} c \psi\left(\lambda x_{1}, \ldots, \lambda x_{k}\right) \\
& \leq c M \psi\left(x_{1}, \ldots, x_{k}\right) .
\end{aligned}
$$

Thus $d(J g, J h) \leq c M$. Hence $d(J g, J h) \leq M d(g, h)$ for all $g, h \in X$, and so $J$ is a strictly contractive mapping on $X$ with Lipschitz constant $M$.

Proposition 3.3. Let $E$ be a linear space, and let $\left(\left(F^{n},\|\cdot\|_{n}\right): n \in \mathbb{N}\right)$ be a multiBanach space. Let $L, k \in \mathbb{N}$ with $L \geq 2$, and let there exist $0 \leq M_{0}<1$ and a function $\varphi: E^{2 k} \rightarrow[0, \infty)$ satisfying

$$
\varphi\left(L x_{1}, L y_{1}, \ldots, L x_{k}, L y_{k}\right) \leq L M_{0} \varphi\left(x_{1}, y_{1} \ldots, x_{k}, y_{k}\right)
$$

for all $x_{1}, \ldots, x_{k}, y_{1}, \ldots, y_{k} \in E$. Suppose that $f: E \rightarrow F$ is a mapping with $f(0)=0$ and

$$
\begin{array}{r}
\left\|\left(f\left(\mu x_{1}+\mu y_{1}\right)-\mu f\left(x_{1}\right)-\mu f\left(y_{1}\right), \ldots, f\left(\mu x_{k}+\mu y_{k}\right)-\mu f\left(x_{k}\right)-\mu f\left(y_{k}\right)\right)\right\|_{k} \\
\leq \varphi\left(x_{1}, y_{1}, \ldots, x_{k}, y_{k}\right)
\end{array}
$$

for all $\mu \in \mathbb{T}$ and $x_{1}, \ldots, x_{k}, y_{1}, \ldots, y_{k} \in E$. Then there exists a unique linear mapping $T: E \rightarrow F$ such that

$$
\left\|\left(f\left(x_{1}\right)-T x_{1}, \ldots, f\left(x_{k}\right)-T x_{k}\right)\right\|_{k} \leq \frac{M_{0}}{1-M_{0}} \psi\left(x_{1}, \ldots, x_{k}\right),
$$


where

$$
\psi\left(x_{1}, \ldots, x_{k}\right):=\sum_{j=1}^{L-1} \varphi\left(j x_{1} / L, x_{1} / L, j x_{2} / L, x_{2} / L, \ldots, j x_{k} / L, x_{k} / L\right)
$$

for all $x_{1}, \ldots, x_{k} \in E$.

Proof. Let $x_{1}, \ldots, x_{k} \in E$. Setting $\mu=1, y_{1}=x_{1}, \ldots, y_{k}=x_{k}$ in (3.2), we obtain

$$
\left\|\left(f\left(2 x_{1}\right)-2 f\left(x_{1}\right), \ldots, f\left(2 x_{k}\right)-2 f\left(x_{k}\right)\right)\right\|_{k} \leq \varphi\left(x_{1}, x_{1}, x_{2}, x_{2}, \ldots, x_{k}, x_{k}\right) .
$$

By using induction one can easily see that

$$
\left\|\left(f\left(L x_{1}\right)-L f\left(x_{1}\right), \ldots, f\left(L x_{k}\right)-L f\left(x_{k}\right)\right)\right\|_{k} \leq \psi\left(L x_{1}, \ldots, L x_{k}\right) .
$$

It follows from (3.1) that $\psi\left(L x_{1}, \ldots, L x_{k}\right) \leq L M_{0} \psi\left(x_{1}, \ldots, x_{k}\right)$, and so, by (3.4), we have

$$
\begin{aligned}
\left\|\left(\frac{1}{L} f\left(L x_{1}\right)-f\left(x_{1}\right), \ldots, \frac{1}{L} f\left(L x_{k}\right)-f\left(x_{k}\right)\right)\right\|_{k} & \leq \frac{1}{L} \psi\left(L x_{1}, \ldots, L x_{k}\right) \\
& \leq M_{0} \psi\left(x_{1}, \ldots, x_{k}\right) .
\end{aligned}
$$

Let $X:=\{g: E \rightarrow F: g(0)=0\}$ and $d$ be as in Lemma 3.2, with $M$ and $\lambda$ replaced by $M_{0}$ and $L$, respectively. Define the mapping $J_{0}: X \rightarrow X$ by

$$
\left(J_{0} g\right)(x):=g(L x) / L \quad(x \in X) .
$$

Then $J_{0}$ is a strictly contractive mapping. By (3.5), we have $d\left(f, J_{0} f\right) \leq M_{0}<\infty$. By Theorem 3.1 (with $f$ for $x$ ), there exists $n_{0} \in \mathbb{N}$ such that the sequence $\left(J_{0}^{n} f\right)$ converges to a fixed point $T$ of $J_{0}$ (so that $T(L x)=L T(x) \quad(x \in E)$ ), $T$ is the unique fixed point of $J_{0}$ in the set $U_{0}=\left\{g \in X: d\left(J_{0}^{n_{0}} f, g\right)<\infty\right\}$, and

$$
d(g, T) \leq d\left(g, J_{0} f\right) /\left(1-M_{0}\right) \quad\left(g \in U_{0}\right)
$$

Since $\lim _{n \rightarrow \infty} d\left(J_{0}^{n} f, T\right)=0$, we easily conclude that

$$
\lim _{n \rightarrow \infty} \frac{f\left(L^{n} x\right)}{L^{n}}=T(x) \quad(x \in E),
$$

where we are using property (a). Clearly $d\left(f, J_{0}^{n_{0}}\right)<\infty$, and so $f \in U_{0}$. Hence

$$
d(f, T) \leq \frac{1}{1-M_{0}} d\left(f, J_{0} f\right) \leq \frac{M_{0}}{1-M_{0}}
$$

and so

$$
\left\|\left(f\left(x_{1}\right)-T\left(x_{1}\right), \ldots, f\left(x_{k}\right)-T\left(x_{k}\right)\right)\right\|_{k} \leq \frac{M_{0}}{1-M_{0}} \psi\left(x_{1}, \ldots, x_{k}\right) \quad\left(x_{1}, \ldots, x_{k} \in E\right) .
$$

Fix $x, y \in E$ and $\mu \in \mathbb{T}$. Let us replace all $x_{1}, \ldots, x_{k}$ by $L^{n} x$ and all $y_{1}, \ldots, y_{k}$ by $L^{n} y$ in (3.2), divide both sides by $L^{n}$, and pass to the limit as $n \rightarrow \infty$. Then, using 
property (a) again, we obtain

$$
\begin{aligned}
\|T(\mu x+\mu y)-\mu T(x)-\mu T(y)\| & \leq \limsup _{n \rightarrow \infty} \frac{1}{L^{n}} \varphi\left(L^{n} x, L^{n} y, \ldots, L^{n} x, L^{n} y\right) \\
& \leq \limsup _{n \rightarrow \infty} M_{0}^{n} \varphi(x, y, \ldots, x, y)=0 .
\end{aligned}
$$

Thus $T(\mu x+\mu y)=\mu T(x)+\mu T(y)$ for all $\mu \in \mathbb{T}$ and $x, y \in E$.

Next, let $\xi \in \mathbb{C}$, and take $K \in \mathbb{N}$ with $K>|\xi|$. By an easy geometric argument, one can see that there are $\mu_{1}, \mu_{2} \in \mathbb{T}$ such that $2 \xi=K\left(\mu_{1}+\mu_{2}\right)$. Using the additivity of $T$, we obtain

$$
\begin{aligned}
T(\xi x) & =T\left(\frac{K}{2} \cdot 2 \cdot \frac{\xi}{K} x\right)=K T\left(\frac{1}{2} \cdot 2 \cdot \frac{\xi}{K} x\right)=\frac{K}{2} T\left(2 \cdot \frac{\xi}{K} x\right) \\
& =\frac{K}{2} T\left(\mu_{1} x+\mu_{2} x\right)=\frac{K}{2}\left(T\left(\mu_{1} x\right)+T\left(\mu_{2} x\right)\right) \\
& =\frac{K}{2}\left(\mu_{1}+\mu_{2}\right) T(x)=\frac{K}{2} \cdot 2 \cdot \frac{\xi}{K}=\xi T(x)
\end{aligned}
$$

for all $x \in E$. Thus the mapping $T$ is linear.

If $S$ is another linear mapping satisfying (3.3), then $S(L x)=L S(x)$ and so $S$ is a fixed point of $J_{0}$. Moreover,

$$
\left\|\left(\frac{f\left(L^{n_{0}} x_{1}\right)}{L^{n_{0}}}-S\left(x_{1}\right), \ldots, \frac{f\left(L^{n_{0}} x_{k}\right)}{L^{n_{0}}}-S\left(x_{k}\right)\right)\right\|_{k} \leq \frac{M_{0}}{1-M_{0}} \psi\left(x_{1}, \ldots, x_{k}\right)
$$

for all $x_{1}, \ldots, x_{k} \in E$. Hence $S$ is a fixed point of $J_{0}$ in the set $U_{0}$, and so $T=S$.

COROLlaRY 3.4. Let $(E,\|\cdot\|)$ be a normed space, and let $\left(\left(F^{n},\|\cdot\|_{n}\right): n \in \mathbb{N}\right)$ be a multi-Banach space. Let $k \in \mathbb{N}, p \in[0,1), \alpha, \beta \geq 0$, and let $f: E \rightarrow F$ be a mapping satisfying $f(0)=0$ and

$$
\begin{array}{r}
\left\|\left(f\left(\mu x_{1}+\mu y_{1}\right)-\mu f\left(x_{1}\right)-\mu f\left(y_{1}\right), \ldots, f\left(\mu x_{k}+\mu y_{k}\right)-\mu f\left(x_{k}\right)-\mu f\left(y_{k}\right)\right)\right\|_{k} \\
\leq \alpha+\beta\left(\left\|x_{1}\right\|^{p}+\left\|y_{1}\right\|^{p}+\cdots+\left\|x_{k}\right\|^{p}+\left\|y_{k}\right\|^{p}\right)
\end{array}
$$

for all $\mu \in \mathbb{T}$, and $x_{1}, \ldots, x_{k}, y_{1}, \ldots, y_{k} \in E$. Then there exists a unique linear mapping $T: E \rightarrow F$ such that

$$
\left\|\left(f\left(x_{1}\right)-T x_{1}, \ldots, f\left(x_{k}\right)-T x_{k}\right)\right\|_{k} \leq \frac{2^{p-1} \alpha+\beta\left(\left\|x_{1}\right\|^{p}+\cdots+\left\|x_{k}\right\|^{p}\right)}{1-2^{p-1}}
$$

for all $x_{1}, \ldots, x_{k} \in E$.

Proof. Set $L=2, M_{0}=2^{p-1}<1$, and

$$
\varphi\left(x_{1}, y_{1}, \ldots, x_{k}, y_{k}\right)=\alpha+\beta\left(\left\|x_{1}\right\|^{p}+\left\|y_{1}\right\|^{p}+\cdots+\left\|x_{k}\right\|^{p}+\left\|y_{k}\right\|^{p}\right),
$$

so that $\varphi$ satisfies (3.1) in Proposition 3.3. We have

$$
\psi\left(x_{1}, \ldots, x_{k}\right)=\alpha+2^{1-p} \beta\left(\left\|x_{1}\right\|^{p}+\cdots+\left\|x_{k}\right\|^{p}\right) \quad\left(x_{1}, \ldots, x_{k} \in E\right),
$$

and so there exists a linear mapping $T$ with the required property.

The following result is our main theorem. 
THEOREM 3.5. Let $\left(\left(E^{n},\|\cdot\|_{n}\right): n \in \mathbb{N}\right)$ be a multi-normed space, let $\left(\left(F^{n},\|\cdot\|_{n}\right)\right.$ : $n \in \mathbb{N})$ be a multi-Banach space, let $\alpha \geq 0$, and let $f: E \rightarrow F$ be a mapping satisfying $f(0)=0$ and

$$
\left\|\left(f\left(\mu x_{1}+\mu y_{1}\right)-\mu f\left(x_{1}\right)-\mu f\left(y_{1}\right), \ldots, f\left(\mu x_{k}+\mu y_{k}\right)-\mu f\left(x_{k}\right)-\mu f\left(y_{k}\right)\right)\right\|_{k} \leq \alpha
$$

for all $k \in \mathbb{N}, \mu \in \mathbb{T}$ and $x_{1}, \ldots, x_{k}, y_{1}, \ldots, y_{k} \in E$. Suppose that $f$ is multi-continuous at some point of $E$. Then there exists a unique multi-bounded linear mapping $T: E \rightarrow F$ such that

$$
\|f(x)-T x\| \leq \alpha
$$

for all $x \in E$.

Proof. By Corollary 3.4 with $p=\beta=0$, for each $k \in \mathbb{N}$, there exists a unique linear mapping $T_{k}: E \rightarrow F$ such that

$$
\left\|\left(f\left(x_{1}\right)-T_{k} x_{1}, \ldots, f\left(x_{k}\right)-T_{k} x_{k}\right)\right\|_{k} \leq \alpha \quad\left(x_{1}, \ldots, x_{k} \in E\right) .
$$

By (A3), in fact $T_{k}=T_{1}(k \in \mathbb{N})$, say $T=T_{1}$. Therefore $\|f(x)-T x\| \leq \alpha \quad(x \in E)$.

We shall prove that $T$ is multi-continuous. Indeed, suppose that $f$ is multicontinuous at $x_{0} \in E$, and assume towards a contradiction that $T$ is not multicontinuous at 0 . Then there exist a sequence $\left(x_{n}\right)$ in $E$ and $\eta>0$ with $\operatorname{Lim}_{n \rightarrow \infty} x_{n}=0$ and

$$
\limsup _{n \rightarrow \infty} \sup _{k}\left\|\left(T x_{n}, \ldots, T x_{n+k-1}\right)\right\|_{k}>\eta
$$

Set $\varepsilon>2 \alpha / \eta$. Then

$$
\limsup _{n \rightarrow \infty} \sup _{k}\left\|\left(T\left(\varepsilon x_{n}+x_{0}\right)-T\left(x_{0}\right), \ldots, T\left(\varepsilon x_{n+k-1}+x_{0}\right)-T\left(x_{0}\right)\right)\right\|_{k}>2 \alpha .
$$

For $n \in \mathbb{N}$, we have

$$
\begin{aligned}
\sup _{k} \|\left(T\left(\varepsilon x_{n}+x_{0}\right)-\right. & \left.T\left(x_{0}\right), \ldots, T\left(\varepsilon x_{n+k-1}+x_{0}\right)-T\left(x_{0}\right)\right) \|_{k} \\
\leq & \sup _{k} \|\left(T\left(\varepsilon x_{n}+x_{0}\right)-f\left(\varepsilon x_{n}+x_{0}\right),\right. \\
& \left.\ldots, T\left(\varepsilon x_{n+k-1}+x_{0}\right)-f\left(\varepsilon x_{n+k-1}+x_{0}\right)\right) \|_{k} \\
& +\sup _{k}\left\|\left(f\left(\varepsilon x_{n}+x_{0}\right)-f\left(x_{0}\right), \ldots, f\left(\varepsilon x_{n+k-1}+x_{0}\right)-f\left(x_{0}\right)\right)\right\|_{k} \\
& +\left\|\left(f\left(x_{0}\right)-T\left(x_{0}\right), \ldots, f\left(x_{0}\right)-T\left(x_{0}\right)\right)\right\|_{k} .
\end{aligned}
$$

Since $\operatorname{Lim}_{n \rightarrow \infty} f\left(\varepsilon x_{n}+x_{0}\right)=f\left(x_{0}\right)$, we have

$$
\limsup _{n \rightarrow \infty} \sup _{k}\left\|\left(T\left(\varepsilon x_{n}+x_{0}\right)-T\left(x_{0}\right), \ldots, T\left(\varepsilon x_{n+k-1}+x_{0}\right)-T\left(x_{0}\right)\right)\right\|_{k} \leq 2 \alpha,
$$

a contradiction. Hence $T$ is multi-continuous. By Theorem 2.8, $T$ is multibounded.

Proposition 3.6. Let $E$ be a linear space, and let $\left(\left(F^{n},\|\cdot\|_{n}\right): n \in \mathbb{N}\right)$ be a multiBanach space. Let $L, k \in \mathbb{N}$ with $L \geq 2$, and let there exist $0 \leq M_{1}<1$ and a function 
$\varphi: E^{2 k} \rightarrow[0, \infty)$ satisfying

$$
\varphi\left(x_{1}, y_{1} \ldots, x_{k}, y_{k}\right) \leq \frac{1}{L} M_{1} \varphi\left(L x_{1}, L y_{1}, \ldots, L x_{k}, L y_{k}\right)
$$

for all $x_{1}, \ldots, x_{k}, y_{1}, \ldots, y_{k} \in E$. Suppose that $f: E \rightarrow F$ is a mapping with $f(0)=0$ and

$$
\begin{array}{r}
\left\|\left(f\left(\mu x_{1}+\mu y_{1}\right)-\mu f\left(x_{1}\right)-\mu f\left(y_{1}\right), \ldots, f\left(\mu x_{k}+\mu y_{k}\right)-\mu f\left(x_{k}\right)-\mu f\left(y_{k}\right)\right)\right\|_{k} \\
\leq \varphi\left(x_{1}, y_{1}, \ldots, x_{k}, y_{k}\right)
\end{array}
$$

for $\mu=1$, i and for all $x_{1}, \ldots, x_{k}, y_{1}, \ldots, y_{k} \in E$. Further, suppose that, for each fixed $x \in E$, the function $t \mapsto f(t x)$ is continuous on $\mathbb{R}$. Then there exists a unique linear mapping $T: E \rightarrow F$ such that

$$
\left\|\left(f\left(x_{1}\right)-T x_{1}, \ldots, f\left(x_{k}\right)-T x_{k}\right)\right\|_{k} \leq \frac{1}{1-M_{1}} \psi\left(x_{1}, \ldots, x_{k}\right)
$$

where

$$
\psi\left(x_{1}, \ldots, x_{k}\right):=\sum_{j=1}^{L-1} \varphi\left(j x_{1} / L, x_{1} / L, j x_{2} / L, x_{2} / L, \ldots, j x_{k} / L, x_{k} / L\right)
$$

for all $x_{1}, \ldots, x_{k} \in E$.

Proof. Let $x_{1}, \ldots, x_{k} \in E$. Setting $\mu=1, y_{1}=x_{1}, \ldots, y_{k}=x_{k}$ in (3.6), we obtain

$$
\left\|\left(f\left(2 x_{1}\right)-2 f\left(x_{1}\right), \ldots, f\left(2 x_{k}\right)-2 f\left(x_{k}\right)\right)\right\|_{k} \leq \varphi\left(x_{1}, x_{1}, x_{2}, x_{2}, \ldots, x_{k}, x_{k}\right) .
$$

By using induction, one can see that

$$
\begin{aligned}
\|\left(f\left(L x_{1}\right)-L f\left(x_{1}\right),\right. & \left.\ldots, f\left(L x_{k}\right)-L f\left(x_{k}\right)\right) \|_{k} \\
& \leq \sum_{j=1}^{L-1} \varphi\left(j x_{1}, x_{1}, j x_{2}, x_{2}, \ldots, j x_{k}, x_{k}\right) .
\end{aligned}
$$

Replacing $x_{j}(1 \leq j \leq k)$ by $x_{j} / L$ in (3.8), we obtain

$$
\left\|\left(f\left(x_{1}\right)-L f\left(x_{1} / L\right), \ldots, f\left(x_{k}\right)-L f\left(x_{k} / L\right)\right)\right\|_{k} \leq \psi\left(x_{1}, \ldots, x_{k}\right) .
$$

Let $X:=\{g: E \rightarrow F: g(0)=0\}$ and $d$ be as in Lemma 3.2, with $M$ and $\lambda$ replacing $M_{0}$ and $1 / L$, respectively. Define the mapping $J_{1}: X \rightarrow X$ by $\left(J_{1} g\right)(x):=\operatorname{Lg}(x / L)$. Then $J_{1}$ is a strictly contractive mapping. By (3.9), we have $d\left(f, J_{1} f\right) \leq 1<\infty$. Essentially, as in the proof of Proposition 3.3, we see that there exists a mapping $T: E \rightarrow F$ such that $T(\mu x+\mu y)=\mu T(x)+\mu T(y)$ for all $x, y \in E$ and $\mu=1$, i, and that $T$ is unique among maps satisfying (3.7).

Now we use the strategy of [19] to show that $T$ is linear. Fix $x_{0} \in E$ and $\rho \in F^{\prime}$. Then the mapping $\Phi: \mathbb{R} \rightarrow \mathbb{R}$ defined by $\Phi(t):=\rho\left(T\left(t x_{0}\right)\right)=\lim _{n \rightarrow \infty} L^{n} \rho\left(f\left(L^{-n} t x_{0}\right)\right)$ is additive. Since $\Phi$ is the pointwise limit of a sequence of continuous functions $L^{n} \rho\left(f\left(L^{-n} t x_{0}\right)\right)$, it is measurable. Thus the additive mapping $\Phi$ is continuous, and hence $\mathbb{R}$-linear. For all $\xi=a_{1}+\mathrm{i} a_{2} \in \mathbb{C}$, where $a_{1}, a_{2} \in \mathbb{R}$, we then have

$$
T(\xi x)=T\left(a_{1} x\right)+\mathrm{i} T\left(a_{2} x\right)=a_{1} T(x)+\mathrm{i} a_{2} T(x)=\xi T(x) .
$$

Therefore $T$ is a linear mapping. 
Corollary 3.7. Let $(E,\|\cdot\|)$ be a normed space and let $\left(\left(F^{n},\|\cdot\|_{n}\right): n \in \mathbb{N}\right)$ be a multi-Banach space. Let $k \in \mathbb{N}, p>1, \beta \geq 0$, and let $f: E \rightarrow F$ be a mapping satisfying $f(0)=0$ and

$$
\begin{array}{r}
\left\|\left(f\left(\mu x_{1}+\mu y_{1}\right)-\mu f\left(x_{1}\right)-\mu f\left(y_{1}\right), \ldots, f\left(\mu x_{k}+\mu y_{k}\right)-\mu f\left(x_{k}\right)-\mu f\left(y_{k}\right)\right)\right\|_{k} \\
\leq \beta\left(\left\|x_{1}\right\|^{p}+\left\|y_{1}\right\|^{p}+\cdots+\left\|x_{k}\right\|^{p}+\left\|y_{k}\right\|^{p}\right)
\end{array}
$$

for $\mu=1$, i and for all $x_{1}, \ldots, x_{k}, y_{1}, \ldots, y_{k} \in E$. If for each fixed $x \in E$ the function $t \mapsto f(t x)$ is continuous on $\mathbb{R}$, then there exists a unique linear mapping $T: E \rightarrow F$ such that

$$
\left\|\left(f\left(x_{1}\right)-T\left(x_{1}\right), \ldots, f\left(x_{k}\right)-T\left(x_{k}\right)\right)\right\|_{k} \leq \frac{\beta}{2^{p-1}-1}\left(\left\|x_{1}\right\|^{p}+\cdots+\left\|x_{k}\right\|^{p}\right)
$$

for all $x_{1}, \ldots, x_{k} \in E$.

Proof. Set

$$
\varphi\left(x_{1}, y_{1}, \ldots, x_{k}, y_{k}\right)=\beta\left(\left\|x_{1}\right\|^{p}+\left\|y_{1}\right\|^{p}+\cdots+\left\|x_{k}\right\|^{p}+\left\|y_{k}\right\|^{p}\right),
$$

and let $M_{1}=2^{1-p}<1$ in Proposition 3.6. Then

$$
\psi\left(x_{1}, \ldots, x_{k}\right)=2^{1-p} \beta\left(\left\|x_{1}\right\|^{p}+\cdots+\left\|x_{k}\right\|^{p}\right) \quad\left(x_{1}, \ldots, x_{k} \in E\right),
$$

and there exists a unique linear mapping $T$ with the required property.

ACKNOWLEDGEMENTS. This work was written whilst the second author was visiting the University of Leeds during his sabbatical leave in 2006; he would like to thank sincerely Professor H. G. Dales for his warm hospitality and members of the School of Mathematics at Leeds for their kindness.

\section{REFERENCES}

1. J. Aczél, A short course on functional equations (D. Reidel Publ. Co., Dordrecht, 1987).

2. M. Amyari and M. S. Moslehian, Approximately ternary semigroup homomorphisms, Lett. Math. Phys. 77 (2006), 1-9.

3. C. Baak and M. S. Moslehian, Stability of $J^{*}$-homomorphisms, Nonlinear Anal. 63 (2005), 42-48.

4. L. Cădariu and V. Radu, Fixed points and the stability of Jensen's functional equation, J. Inequal. Pure Appl. Math. 4 (2003), no. 1, art. 4, 7 pp. 2002).

5. S. Czerwik, Functional equations and inequalities in several variables (World Scientific, (preprint).

6. H. G. Dales and M. E. Polyakov, Multi-normed spaces and multi-Banach algebras 431-434.

7. Z. Gajda, On stability of additive mappings, Internat. J. Math. Math. Sci. 14 (1991),

8. P. Găvruta, A generalization of the Hyers-Ulam-Rassias stability of approximately additive mappings, J. Math. Anal. Appl. 184 (1994), 431-436.

9. D. H. Hyers, On the stability of the linear functional equation, Proc. Nat. Acad. Sci. U.S.A. 27 (1941), 222-224.

10. D. H. Hyers, G. Isac and Th. M. Rassias, Stability of functional equations in several variables (Birkhäuser, Basel, 1998).

11. G. Isac and Th. M. Rassias, Stability of $\psi$-additive mappings: Applications to nonlinear analysis, Internat. J. Math. Math. Sci. 19 (1996), 219-228. 
12. K. Jarosz, Perturbations of Banach algebras, Lecture Notes in Mathematics, No. 1120 (Springer-Verlag, 1985).

13. B. E. Johnson, Approximately multiplicative functionals, J. London Math. Soc. (2) 34 (1986), 489-510.

14. B. E. Johnson, Approximately multiplicative maps between Banach algebras, J. London Math. Soc. (2) 37 (1988), 294-316.

15. S.-M. Jung, Hyers-Ulam-Rassias stability of functional equations in mathematical analysis (Hadronic Press, Palm Harbor, Florida, 2001).

16. B. Margolis and J. B. Diaz, A fixed point theorem of the alternative for contractions on a generalized complete metric space, Bull. Amer. Math. Soc. 126 (1968), 305-309.

17. M. Mirzavaziri and M. S. Moslehian, A fixed point approach to stability of a quadratic equation, Bull. Brazilian Math. Soc. 37 (2006), 361-376.

18. M. S. Moslehian, Approximately vanishing of topological cohomology groups, J. Math. Anal. Appl. 318 (2006), 758-771.

19. Th. M. Rassias, On the stability of the linear mapping in Banach spaces, Proc. Amer. Math. Soc. 72 (1978), 297-300.

20. Th. M. Rassias, Problem 16, Second report of the 27th International Symposium on Functional Equations, Aequationes Math. 39 (1990), 292-293.

21. Th. M. Rassias and P. Šemrl, On the behaviour of mappings which do not satisfy Hyers-Ulam stability, Proc. Amer. Math. Soc. 114 (1992), 989-993.

22. S. M. Ulam, Problems in modern mathematics, Chapter VI (Wiley, 1964). 